Chirurg 2019 $\cdot 90: 324$

https://doi.org/10.1007/s00104-019-0808-5

Online publiziert: 6. Februar 2019

(c) Springer Medizin Verlag GmbH, ein Teil von Springer Nature 2019

CrossMark

G. Schlager $\cdot$ M. Schrempf $\cdot$ M. Anthuber

Klinik für Allgemein-, Viszeral- Transplantationschirurgie, Klinikum Augsburg, Augsburg, Deutschland

\title{
Präoperative Diät reduziert den Blutverlust bei Leberteilresektionen
}

vs. $1991 \mathrm{kcal}, 19$ vs. $70 \mathrm{~g}$ Fett, jeweils $p<0,0001)$. In dieser Gruppe fand sich im Mittel ein signifikant geringerer intraoperativer Blutverlust (452 vs. $863 \mathrm{ml}$, $p=0,02$ ). Verglichen mit der Kontrollgruppe führte dies zu einer tendenziell geringeren Bluttransfusionsmenge pro Patient (138 vs. $322 \mathrm{ml}, p=0,06$ ). Die Operateure empfanden die Leber in der Diätgruppe leichter zu mobilisieren, was sich in einem niedrigeren Mittelwert des Ease of Mobility Score widerspiegelte $(1,8$ vs. $2,9, p=0,004,1=$ einfach, $5=$ schwierig). Hinsichtlich der Dauer des Krankenhausaufenthaltes und der Komplikationsrate (Gallenleckage, Abszess u.a.) gab es keine Unterschiede. Der Glykogengehalt der Hepatozyten war signifikant geringer in der Diätgruppe $(p<0,0001)$. Es zeigte sich jedoch kein Unterschied in der Anzahl der Patienten mit Steatosis hepatis oder Steatohepatitis.

Diskussion und Fazit. Die methodisch hochwertige Studie liefert neue Evidenz für eine Präkonditionierung in der onkologischen Viszeralchirurgie [1]. Eine Diät von nur einer Woche hatte bereits positiven Einfluss auf den intraoperativen Blutverlust. Die Transfusionsmenge innerhalb der Diätgruppe war geringer, wenngleich eine statistische Signifikanz möglicherweise durch die kleine Studienpopulation nicht erreicht wurde. Im Hinblick auf das Vorliegen einer Steatosis hepatis zeigte sich kein Unterschied zwischen den Gruppen. Daher führten die Autoren die bessere Operabilität der Leber auf den geringeren Glykogengehalt und somit Wassergehalt der Hepatozyten in der Diätgruppe zurück. Die Diät zeigte weder Auswirkung auf die perioperative Letalität, die Operationsoder stationäre Verweildauer noch auf die Komplikationsrate. Patienten mit einer präoperativen Anämie wurden nicht gesondert im Sinne einer Subgruppenanalyse verglichen. Hier hätte die Studie wertvolle Informationen liefern können, ob sich die Diät günstig auf die perioperative Morbidität anämischer Patienten auswirkt. Dennoch stellt die kalorienund fettarme Diät einen kostengünstigen und schonenden Ansatz dar, Patienten vor einer onkologischen Leberteilresektion zu präkonditionieren.

\section{Korrespondenzadresse}

Prof. Dr. M. Anthuber

Klinik für Allgemein-, Viszeral- Transplantationschirurgie, Klinikum Augsburg

Stenglinstr. 2, 86156 Augsburg, Deutschland matthias.anthuber@klinikum-augsburg.de

Interessenkonflikt. G. Schlager, M. Schrempf und M. Anthuber geben an, dass kein Interessenkonflikt besteht.

\section{Literatur}

1. Pfirrmann D, Simon P, Mehdorn M et al (2018) Präkonditionierung vor viszeralonkologischen Operationen. Chirurg. https://doi.org/10.1007/ s00104-018-0709-z 